\title{
ICEF2020-2924
}

\section{Parametric Studies of a Novel Combustion Modelling Approach for Low Temperature Diesel Spray Simulation}

\author{
XiaoHang Fang*, Nikola Sekularac ${ }^{\dagger}$ and Martin H. Davy \\ Thermal Propulsion System Research Group \\ Department of Engineering Science \\ University of Oxford \\ Oxford, OX1 3PJ, United Kingdom \\ Email: xiaohang.fang@eng.ox.ac.uk
}

\section{ABSTRACT}

Conditional Source-term Estimation (CSE) is a combustion model based on the conditional moment hypothesis where transport equations for reactive species are conditionally averaged on conserved scalars. Major advantages of this strategy are the reduced spatial dependence of the conditional averages and negligible fluctuations around the conditional averages, which considerably simplify the reaction rate closure. Historically, simulations using CSE are limited to low carbon fuels (i.e. methane and hydrogen) where the reduced chemistry manifold can be constructed through techniques including intrinsic low dimensional manifolds and trajectory generated manifolds. However, the use of such strategies to create manifolds for diesel surrogates has proven problematic.

In this study, the potential of a combination of an unsteady Flamelet Generated Manifold (FGM) and the Conditional Source-term Estimation approach to predict the ignition and flame propagation on an autoigniting $n$-dodecane spray flame is assessed. Simulations are performed on a single-hole injection of n-dodecane under a wide range of Engine Combustion Network's "Spray A" conditions within a Reynolds-averaged Navier-Stokes (RANS) framework. Results from parametric sweeps of ambient temperature and oxygen concentration are qualitatively validated against experimental data from the literature and compared against predictions from an industry standard well-stirred reactor model. The efficacy of the CSE-FGM RANS approach

\footnotetext{
*Address all correspondence to this author.
}

${ }^{\dagger}$ The authors share equal contribution to the article. in predicting flame characteristics is evaluated and further compared with high fidelity CSE-FGM simulations using the Large Eddy Simulation (LES) turbulence model.

Overall, it was found that the CSE-FGM RANS model was able to capture global flame properties-showing particular strength in predicting auto-ignition events in the low temperature region. The model was also able to satisfactorily capture details of the two-stage ignition process. The results were shown to be consistent with those of the CSE-FGM LES model, demonstrating the adaptability of the CSE-FGM approach to different turbulence modelling paradigms.

\section{NOMENCLATURE}

C

CMC Conditional Moment Closure

CSE Conditional Source-term Estimation

D Diffusion Coefficient

ECN Engine Combustion Network

FGM Flamelet Generated Manifold Model

IDT Ignition Delay Time

KH-RT Kelvin-Helmholtz Raleigh-Taylor

LES Large Eddy Simulation

LOL Lift-Off Length

RANS Reynolds Averaged Navier-Stokes

TCI Turbulence Chemistry Interaction

WSR Well Stirred Reactor Model

$Z \quad$ Mixture Fraction 


\section{INTRODUCTION}

Modern compression ignition engines are characterised by the use of low temperature combustion strategies within selected regions of the operating map for the reduction of particulate matter and $\mathrm{NO}_{x}$ emissions. This has prompted numerous investigations into the detailed in-cylinder processes of low temperature diesel combustion [?,?]. These range from fuel spray interaction/behaviour to chemical kinetics of the charge and other related physical phenomena. Diesel combustion is a complex process due to its inherent heterogeneity where, liquid fuel breakup, atomization, vaporisation, fuel-air mixing, burned-unburned gas mixing and combustion occur simultaneously. Hence, there are a multitude of physical and chemical processes that contribute to the combustion phasing and pollutant formation.

Numerical modelling has become an increasingly popular research tool in recent years as dramatic increases in computational power that have enabled the use of advanced computational fluid dynamics models to describe many of the complex phenomena occurring during diesel combustion. As part of a major international collaboration among experimental and computational researchers within the engine combustion community, a characteristic diesel spray, "Spray A", has been defined for the study. This flame can be described as a lifted flame composed of both rich partially premixed region and a diffusion flame. In order to accurately simulate the spray flame, two main aspects are considered: chemical mechanisms that describe the oxidation of diesel fuel and turbulence chemistry interactions (TCI) accounting for how fluctuations in the chemical source terms and the turbulent flow are mutually affected under both Reynolds Averaged Navier-Stokes (RANS) and Large Eddy Simulation (LES) frameworks.

Various studies have explored the effects of different chemical mechanisms in predicting diesel combustion, both generally and with specific reference to "Spray A" [?,?]. The autoignition of a diesel spray is recognized as a multi-stage combustion event, where high temperature and low temperature chemistry are separated by a cool flame. Accurate low temperature chemistry is therefore essential in order to predict the autoignition process and the high temperature combustion where major species of combustion are generated $[?, ?]$.

In terms of being able to accurately represent the TCI, the choice of combustion model is key. Studies have suggested that diesel engine combustion simulation requires the use of finite rate chemistry models in solving chemical kinetics [?, ?]. However, the simplest form of finite rate chemistry model, the well-stirred reactor (WSR), does not consider TCI at all. Accordingly, the WSR model is not best suited for spray combustion simulations, especially for low reactive conditions where TCI are known to play a significant role [?]. In contrast, models that inherently incorporate TCI - such as Transported Probability Density Function (TPDF) and Conditional Moment Closure (CMC) methods - have shown promising results predicting diesel spray combus- tion [?, ?]. However, both of these modelling approaches imply a high computational cost, especially when incorporating a large chemical mechanism, making it difficult to perform engine calculations and high fidelity simulations.

An alternative method of considering both detailed chemistry and TCI is through the application of the flamelet concept, whereby a turbulent flame is treated as an ensemble of onedimensional strained flames, combined with a presumed probability density function (PDF). The ability of a variety of flamelet based models to predict low temperature diesel spray combustion is well demonstrated in the literature $[?, ?, ?]$. However, although flamelet methods have been successfully applied to different turbulent flames, challenges have been encountered - especially when flamelet models were used to address multiple combustion regimes (premixed, partially-premixed and diffusion flames) across a wide range of time-scales within a single system. In these cases, different adaptions of the flamelet model are required, involving additional dimensions in the flamelet tables and alternative characteristics flames, which significantly increase the computational cost when detailed chemistry is involved [?,?].

Conditional Source-term Estimation (CSE), which was first proposed by Bushe \& Steiner [?] in 1999, falls within the category of statistical combustion models. The model is based on the experimental observations, where fluctuations in scalar quantities can be significantly reduced after conditioning over a representative quantity [?]. It inherits CMC's advantage of having fewer constraints on combustion regimes but requires less computational resources when higher-order closure or a second conditioning variable is introduced [?]. Although successful implementations have been performed in both premixed and non-premixed flames, most previous studies are focused on methane/air flame due to the complexity of including detailed chemistry within the model.

Recently, in a study of "Spray A" combustion under the standard ECN baseline conditions, the authors demonstrated, for the first time, the potential of a combined CSE and Flamelet Generated Manifold (FGM) approach for the study of complex alkanes [?]. In this referenced work, the CSE-FGM model was shown to be well suited for detailed combustion analysis of diesel spray flames within both RANS and LES frameworks.

The current study presents an extension of this previous work considering further the ability of the CSE-FGM combustion model to predict "Spray A" under quasi-steady and transient conditions. Here we focus particularly on the effects of ambient temperature and oxygen concentration, on the predicted reactivity of the mixture and the spray structure within a RANS simulation. The modelling results are compared with the available experimental data and previous simulations performed using the Well-Stirred Reactor (SAGE) model. This includes a detailed description of trends in ignition delay, the flame lift-off length, and the analysis of flame spatial contours. The autoignition process 
and the reacting spray structure are also discussed in detail.

Finally, in order to assess the adaptability of the CSE-FGM approach to different turbulence models, the flame structure predicted by the RANS model under the baseline ECN conditions using two different chemical mechanisms is compared with the results of previous LES studies. The differences between using RANS and LES are highlighted. An additional evaluation of the capability of CSE in taking into account different chemical mechanisms and turbulence model is given.

\section{COMBUSTION MODEL}

For simulations that are not fully resolved i.e. RANS and LES, obtaining filtered or averaged reaction rates directly from filtered or averaged values of the scalar field can lead to considerable discrepancies compared to fully resolved results. CSE invokes the same first-order hypothesis as CMC for closing chemical source terms. However, whereas the CMC approach is based on solving transport equations for conditional mass fractions and temperature that contain contain unclosed terms, CSE estimates the conditional means from unconditionally averaged scalars thus reducing the computational time required [?]. Adapted to either spatially filtered or averaged equations, respectively such as in LES and RANS, the mean conditional chemical source term for species $i$ is defined as follows:

$$
\overline{\dot{\omega}_{i} \mid \eta} \approx \dot{\omega}_{i}\left(\overline{T \mid \eta}, \overline{Y_{i} \mid \eta}, \overline{\rho \mid \eta}\right)
$$

where $\eta$ is the sample space variable of the mixture fraction $Z$, $\overline{T \mid \eta}$ is the conditional temperature, $\overline{Y_{i} \mid \eta}$ and $\overline{\rho \mid \eta}$ are respectively the conditional mass fraction of species $i$ and density. The unconditional mean chemical source term is obtained by integrating the conditional value presented in the above Equation 1, over the Favre averaged PDF of the mixture fraction, usually defined for non-premixed cases through a $\beta$-function, denoted as $\bar{P}(\eta, \vec{x}, t)$ :

$$
\overline{\dot{\omega}}_{i}(\vec{x}, t)=\int_{\eta}\left\langle\dot{\omega}_{i} \mid \eta\right\rangle(\eta, \vec{x}, t) \bar{P}(\eta, \vec{x}, t) d \eta
$$

The entire solving process of the authors' currently implemented CSE combustion model can be seen in Figure 1. Additionally, and in reference to [?], groups of localized cells referred to as an ensemble are selected in the flow field with statistical homogeneity in conditional averages, enabling the species' mass fractions to be determined as follows:

$$
\widetilde{Y}_{i}\left(\vec{x}_{j}, t\right)=\int_{\eta} \overline{Y_{i} \mid \eta}\left(\eta, \vec{x}_{j}, t\right) \widetilde{P}\left(\eta, \vec{x}_{j}, t\right) d \eta \quad x_{j} \in E
$$

where $\vec{x}_{j}$ is the spatial coordinate of the $j^{t h}$ point in the ensemble $E . \widetilde{Y}_{i}\left(\vec{x}_{j}, t\right)$ are determined via transport equations while
$\overline{Y_{i} \mid \eta}\left(\eta, \vec{x}_{j}, t\right)$ are the unknown values of this problem.

Equation 3, which is a Fredholm integral equation with $\widetilde{P}\left(\eta, \vec{x}_{j}, t\right)$ as the kernel, must be solved at every time step in the reacting flow solver to provide the conditional averages enabling Equation 1 to close the chemical reaction source terms. Further information regarding the calculation of $\overline{Y_{i} \mid \eta}\left(\eta, \vec{x}_{j}, t\right)$ can be found in previous works undertaken by the group, [?] refers.

In order to incorporate detailed chemistry within the CSE framework, a chemistry model is needed during the solving process so that it can accurately predict the complex chemical phenomena occurring in diesel combustion. Trajectory Generated Low-Dimensional Manifolds (TGLDM) [?] have been considered, but studies have shown that this approach might not be appropriate for use with heavy fuels such as diesel surrogates. This has led the authors' to the use of a flamelet based chemistry tabulation method which similarly combines the capability of including complex chemical schemes with relatively low computational costs. The Flamelet Generated Manifold (FGM), presented formally in the section below, has been successfully coupled with CSE, giving satisfactory results against experimental data at baseline "Spray A" conditions [?]. The coupling between the CSE routine and the tabulated chemistry routine, here referring to the FGM, is shown in Figure 1. It is important to note here that the FGM is used within the routine as a chemistry module as opposed to a combustion model.

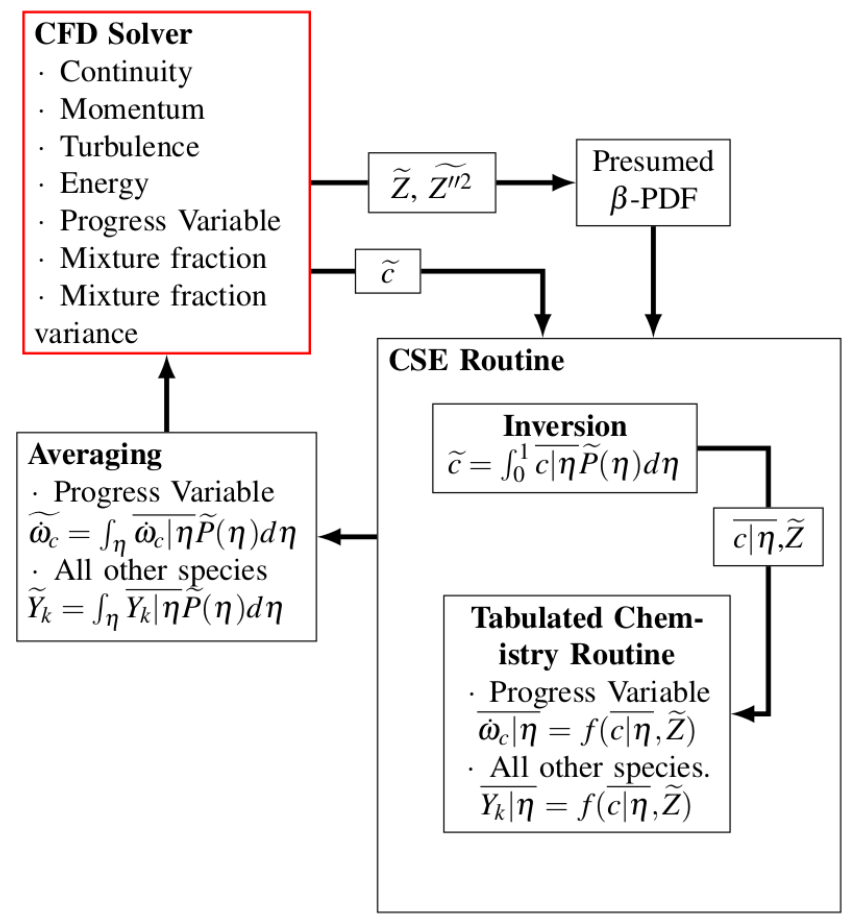

FIGURE 1: CSE Routine Structure for ECN diesel "Spray A" 


\section{FLAMELET GENERATED MANIFOLD}

First proposed by van Oijen and de Goey [?], FGM is a chemistry tabulation method originated from flamelet concepts. It treats a multi-dimensional flame as an ensemble of onedimensional (1D) laminar flames enabling, therefore, the construction of a low-dimensional manifold tabulating reaction rates and species mass fractions as a function of a limited set of coordinates such as the progress variable $C$ (typically used in premixed combustion scenarios) and/or the mixture fraction $Z$ that is mostly employed for non-premixed flames.

\section{Flame configuration}

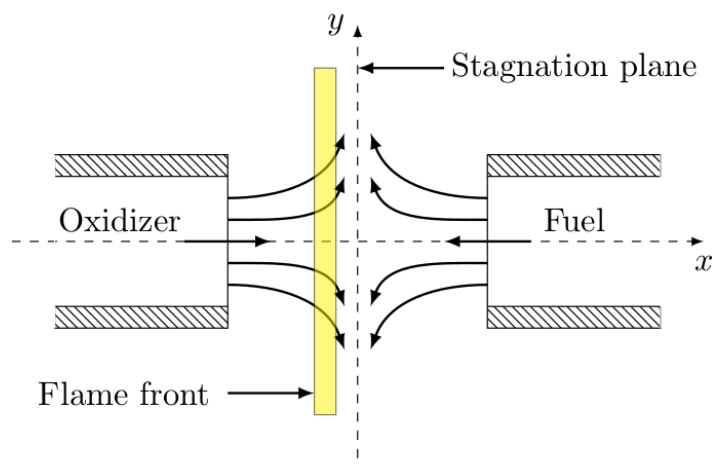

FIGURE 2: Schematic of laminar counter-flow diffusion flame

The FGM method requires that a flame configuration must be first selected to represent the chemical processes of the flame of interest. Diesel combustion is mainly characterised by a nonpremixed regime, and is thus, best represented by the igniting counter-flow diffusion flame (CDF) where the oxidizer and the fuel streams are opposed to each other. The reaction zone is perpendicular to the two flows, as shown in Figure 2, on the oxidizer side of the stagnation plane. Following Stahl and Warnatz [?] one-dimensional governing equations, $\mathrm{CDF}$ formulas are given as:

$$
\begin{gathered}
\frac{\partial \rho}{\partial t}+\frac{\partial \rho U}{\partial x}=-\rho K \\
\frac{\partial \rho h}{\partial t}+\frac{\partial \rho U h}{\partial x}=\frac{\partial}{\partial x}\left(\rho D \frac{\partial h}{\partial x}\right)-\rho K h \\
\frac{\partial \rho Y_{i}}{\partial t}+\frac{\partial \rho U Y_{i}}{\partial x}=\frac{\partial}{\partial x}\left(\rho D \frac{\partial Y_{i}}{\partial x}\right)+\dot{\omega}_{i}-\rho K Y_{i}
\end{gathered}
$$

$$
\frac{\partial \rho K}{\partial t}+\frac{\partial \rho U K}{\partial x}=\frac{\partial}{\partial x}\left(\mu \frac{\partial K}{\partial x}\right)+\rho_{o x} a^{2}-\rho K^{2}
$$

where $D$ assumes an unity Lewis number. $K(x, t)=\frac{\partial u_{y}}{\partial y}$ is the stretch rate or the flame stretch field which is a function of the $x$ coordinate and the applied strain rate $a$ at the oxidizer boundary.

In this work, the CHEM1D code [?] of Eindhoven University of Technology is used to solve one-dimensional CDF in physical space and time with the possibility to choose different chemical mechanisms. In order to account for the unsteadiness in the spray flame, igniting flamelets are included. For each condition tested, a corresponding manifold is needed for the CSE calculation. Figure 3 shows the two different manifolds generated by solving Equations 4-7 for a fixed n-dodecane mechanism (Yao) at a strain rate of $500 \mathrm{~s}^{-1}$, from an initially mixed but nonreacting state (green line) at ambient temperatures and pressures of $800 \mathrm{~K}$ and $5.25 \mathrm{MPa}$ and $1100 \mathrm{~K}$ and 7.3 MPa. The black lines in the figure illustrate the temporal evolution of the laminar flame before reaching a quasi-steady state (red line). Following this, steady-state flamelets are solved down to $1 \mathrm{~s}^{-1}$ to complete the manifold close to chemical equilibrium. Note that the maximum temperature reached by the quasi-steady state solution increases as a function of the ambient conditions, respectively $2100 \mathrm{~K}$ and $2400 \mathrm{~K}$.

\section{Parametrization of the FGM}

The full composition space of the flamelet solutions is described by $N_{s}+2$-dimensional solutions spanned by enthalpy, pressure and $N_{s}$ number of species. These are parametrized against the mixture fraction and the reaction progress variable. Indeed, due to its monotonic function of spatial coordinate $x$, the mixture fraction $Z$ defined through Bilger's definition [?], allows direct mapping of the flamelet solutions from physical space to mixture fraction space. More information regarding the parametrization of the FGM can be found in authors previous works $[?, ?, ?]$.

$$
C=\frac{Y_{\mathrm{CH}_{2} \mathrm{O}}}{Y_{\mathrm{CH}_{2} \mathrm{O}}^{e q}}+\frac{Y_{\mathrm{CO}}}{Y_{\mathrm{CO}}^{e q}}+\frac{Y_{\mathrm{CO}_{2}}}{Y_{\mathrm{CO}_{2}}^{e q}}
$$

In this study, the progress variable $C$ is used as the second reduced controlling variable - here it is defined as a weighted linear combination of certain key species mass fractions that represent each stage of the combustion evolution as per Equation 8. Formaldehyde $\left(\mathrm{CH}_{2} \mathrm{O}\right)$, carbon monoxide and carbon dioxide have been chosen to respectively capture the first stage of ignition [?], the intermediate and end of combustion [?]. Each species is weighted by its equilibrium mass fraction, enabling it to have a balanced contribution. Figure 4 shows the resultant manifolds in 


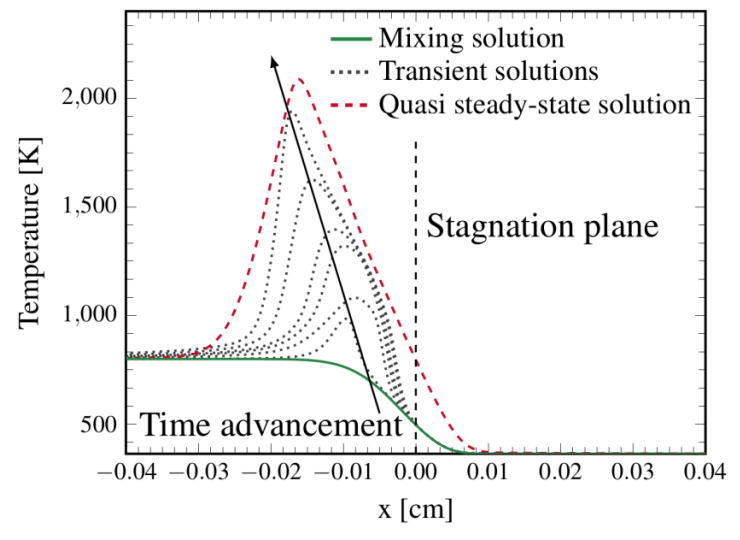

(a) $\mathrm{T}_{a m b}=800 \mathrm{~K}, \mathrm{P}_{a m b}=5.25 \mathrm{MPa}$

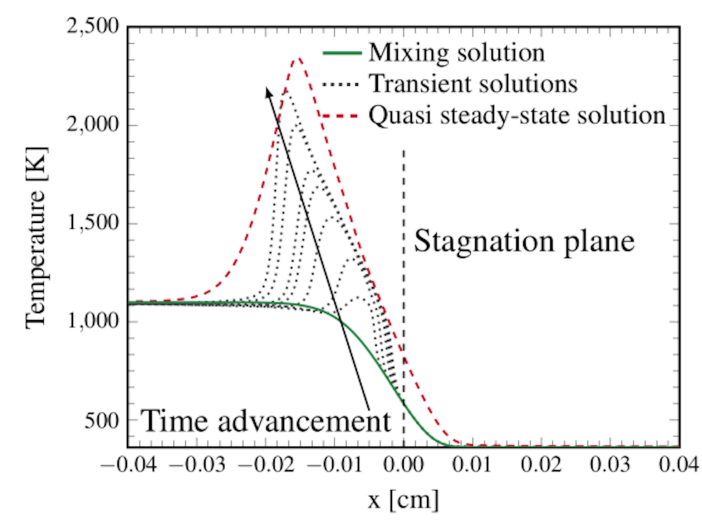

(b) $\mathrm{T}_{a m b}=1100 \mathrm{~K}, \mathrm{P}_{a m b}=7.3 \mathrm{MPa}$

FIGURE 3: Temperature evolution in physical space $x$ and time $t$ for two counter-flow diffusion flames with n-dodecane: $a=500$ $\mathrm{s}^{-1}$ and $\mathrm{T}_{f}=363 \mathrm{~K}$

mixture fraction and progress variable space for the ambient temperatures and pressure pairs of $800 \mathrm{~K}$ and $5.25 \mathrm{MPa}$ and $1100 \mathrm{~K}$ and 7.3 MPa previously depicted with respect to physical space, $x$, and temperature, $T$, in Figure 3. Figure 4 provides significantly greater insight into the nature of the two manifolds. Each of the FGMs is characterised by three well defined reaction zones as expected. The low progress variable values indicate the early ignition stage, while the intermediate and high values of $C$ promote the intermediate and end of combustion respectively. The $800 \mathrm{~K}$ manifold shows relatively lower source term values in all three regions which corresponds to the extended temperature rise in flamelet solutions for this case, illustrated in Figure 3(a). The $1100 \mathrm{~K}$ manifold is characterised by notably higher chemical source term values during the early stage of combustion which is expected to have a significant impact on ignition timing.
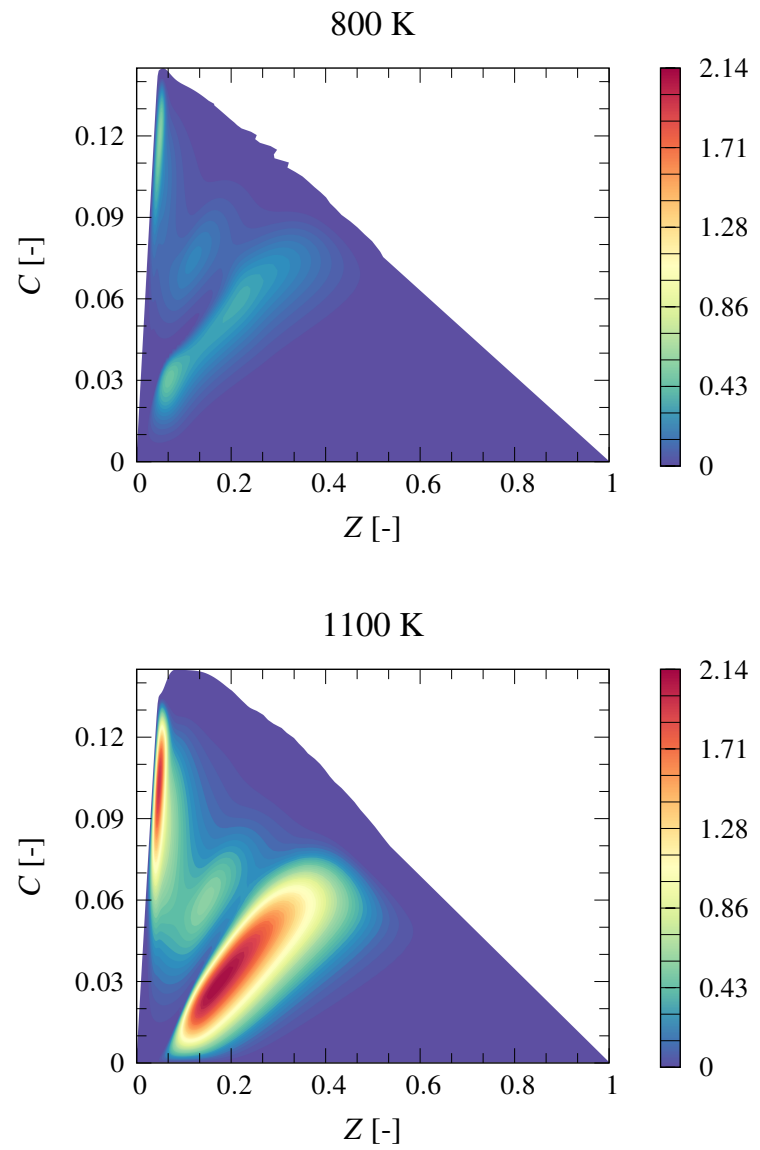

FIGURE 4: Reaction rate of the progress variable for two manifolds with different ambient conditions (Top figure: $800 \mathrm{~K}, 5.25$ $\mathrm{MPa}$, Bottom figure: $1100 \mathrm{~K}, 7.3 \mathrm{MPa})$. The colourbar on the right corresponds to the colourmap of $\dot{\omega}_{c} \cdot 10^{5}$

\section{COMPUTATIONAL SETUP}

A parametric study of the CSE-FGM combustion model has been performed under the Reynolds Averaged Navier-Stokes framework. Simulations of ECN "Spray A" were made across the full range of ambient conditions listed in Table 1, Tests I-VI, and validated against the ECN's high-quality and high-fidelity datasets for both non-reacting and reacting test conditions which can be found at [?]. The CSE-FGM RANS modelling routines have been developed in the commercially available CFD solver CONVERGE through the use of user-defined functions and CONVERGE's in-built capabilities [?]. The Pressure Implicit with Splitting of Operators (PISO) algorithm [?] was used to solve the transport equations along with the Rhie-Chow [?] algorithm to prevent checker-boarding. A second-order accurate central differencing schemes for spatial discretization was applied along with a variable time-step for convection, diffusion and the speed of sound, based on the Courant-Friedrichs-Lewy 


\begin{tabular}{cccc}
\hline Test & $\mathrm{O}_{2}[\%$ volume $]$ & $\mathrm{T}_{a m b}[\mathrm{~K}]$ & Unchanged \\
\hline 0 & 0 & 900 & Fuel $=$ NC12H26 \\
I & 15 & 800 & Fuel mass $=14[\mathrm{mg}]$ \\
II & $\mathbf{1 5}$ & $\mathbf{9 0 0}$ & $\phi_{\text {nozzle }}=0.090[\mathrm{~mm}]$ \\
III & 15 & 1000 & $\rho_{a m b}=22.8\left[\mathrm{kgm}^{-3}\right]$ \\
IV & 15 & 1100 & $\mathrm{~T}_{f u}=363[\mathrm{~K}]$ \\
V & 13 & 900 & $\mathrm{P}_{\text {inj }} 150[\mathrm{MPa}]$ \\
VI & 21 & 900 & \\
\hline
\end{tabular}

TABLE 1: ECN "Spray A" experimental nominal test conditions. "Test II" denotes ECN baseline condition

condition. Turbulence was modelled using the $\mathrm{k}-\varepsilon$ model with modified constants given in authors previous study [?]. Additionally, the well known Lagrangian-Eulerian method was used to model the liquid phase with all sub-models quoted in Table 2 . More detailed spray modelling set-up and sub-model constants can be found in the group's previous publications [?, ?]. The chemical mechanism chosen for the RANS studies is that of Yao et al. [?], which is characterised by 54 species and 268 reactions, subsequently referred to simply as the Yao mechanism.

The computational domain for RANS is set with the injector positioned centrally and an injection flow being in the axial direction. The walls boundaries have been set to as non-slip walls and fixed at a temperature of $461 \mathrm{~K}$, as measured in the experiments.

\begin{aligned} & \hline \multicolumn{2}{c}{ Sub-models } \\ & \hline Injection Blob method \\ & Droplet breakup KH-RT without breakup length \\ & Droplet drag Dynamic droplet drag \\ & Droplet collisions No Time Counter \\ & Vaporisation Frossling correlation \\ & Droplet heat transfer Ranz-Marshall \\ & Turbulent dispersion O'Rourke \\ & \hline\end{aligned}

TABLE 2: Spray simulation discrete phase sub-models

\section{NON-REACTING SPRAY}

A non-reacting spray is first modelled and compared against experimental data before addressing the reacting spray case. The set-up is identical to previous non-reacting simulations performed by the authors [?]. The test conditions for the nonreacting spray study are quoted in Table 1 under the name of "Test 0 ". The liquid and vapour penetrations are studied against the experiment, respectively defined as the axial location at which $95 \%$ of the liquid mass threshold is reached and as the maximum distance from the injector where the fuel mass fraction is equal to $0.1 \%$. The below Figure 5 illustrates the spray model predictions who are in overall in excellent agreement with experimental data, for both liquid and vapour penetrations, with a slight deviation for the vapour penetration until $0.25 \mathrm{~ms}$ ASOI is reached.

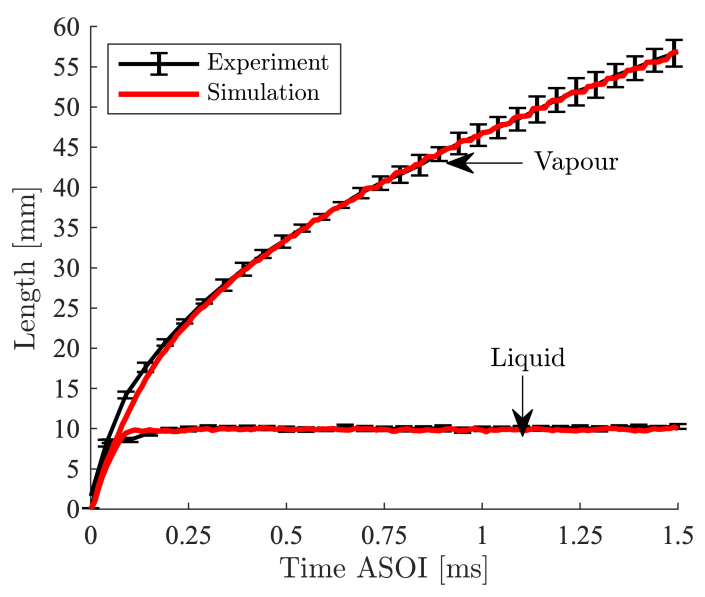

FIGURE 5: Non-reacting liquid and vapor penetration for "Spray A" baseline conditions

\section{REACTING SPRAY RESULTS}

Following the description of the modelling approach, numerical set-up and non-reacting sprays results, this section will discuss and validate the CSE-FGM with across a wide range of ambient boundary conditions. Simulations within the RANS framework will be discussed first in detail. The results are primarily evaluated in terms of the most relevant characteristics of the transient diesel spray, including the autoignition event and the quasi-steady flame structure.

\section{Ambient temperature sweep}

First, the performance of the CSE-FGM combustion model was examined with respect to variations in ambient temperature with all other parameters fixed. The boundary conditions for these tests are given in Table 1, Tests I-IV refer. Note that a new tabulated manifold was generated for each of the conditions listed in the Table. The CSE-FGM results are compared with the results of simulations made using the same spray model in combination with the CONVERGE well-stirred reactor combustion model (SAGE), and also mean experimental results from the $\mathrm{ECN}$. Following the ECN simulation guidelines, the ignition delay time is defined as the first time at which the Favre averaged hydroxyl $(\mathrm{OH})$ mass fraction reaches $2 \%$ of the domain maximum after steady state is established and the lift-off length is 

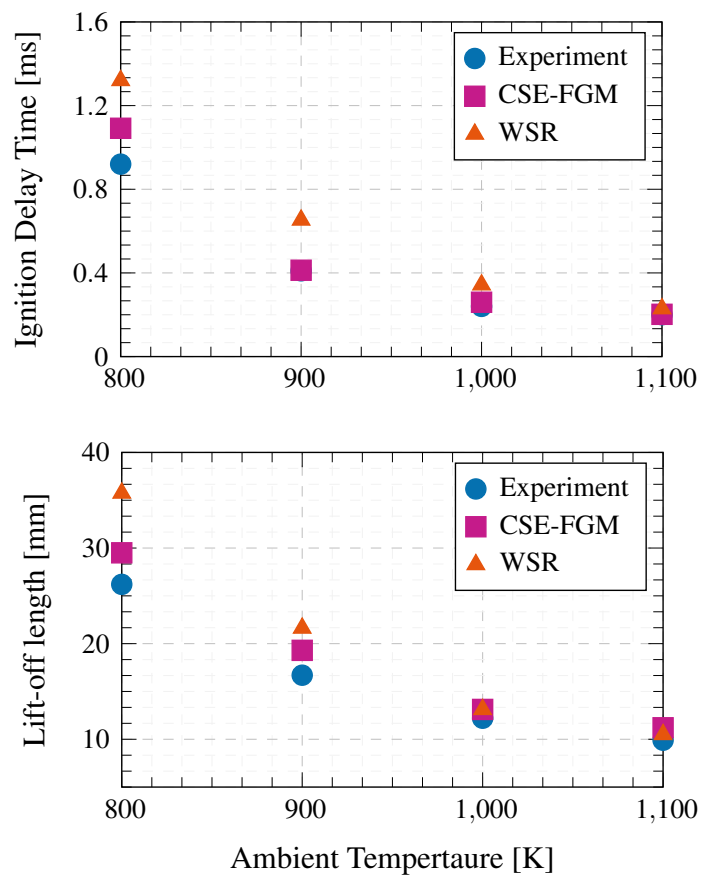

FIGURE 6: Parametric studies of RANS CSE-FGM at different ambient temperature conditions compared against the mean experimental results and well-stirred reactor simulation results for the ignition delay time (top) and the flame lift-off length (bottom)

given as the distance from the injector nozzle at which the Favre averaged $\mathrm{OH}$ mass fraction reaches $14 \%$ of the instantaneous maximum during the steady-state duration.

Figure 6 illustrates the IDT and LOL of the two combustion models against the mean of the experimental data. Both the CSE-FGM and the WSR models capture the general trends in autoignition and lift-off length with temperature. As expected, both the ignition and the lift-off length are increased by lower ambient temperature boundary conditions. In relative terms, the CSEFGM model shows a significant benefit over the WSR, particularly at the baseline and lower temperature conditions where the WSR model substantially overpredicts. This overprediction is attributed to the fact that turbulence chemistry interactions are not included within the WSR model - previous studies have shown the importance of including TCI and sub-grid variance effects in the prediction of low temperature ignition [?,?]. The local inhomogeneity in mixture fracture space and the flow strain effects are found to promote two stage combustion in a low temperature transient diesel spray. The inclusion of TCI in the CSE-FGM model therefore allows it to predict ignition significantly better than the WSR model at lower temperatures.

In order to further study the flame propagation and stabilisation mechanisms, the flame temperature contours and mass fraction distributions of intermediate key species were analysed.
Diesel spray flames are typically characterised by two stage ignition, where studies have found the formation of intermediate species $\mathrm{CH}_{2} \mathrm{O}$ to be a strong indication of first stage ignition [?] and the high temperature second stage ignition is visualised by the formation of $\mathrm{OH}$ radicals.

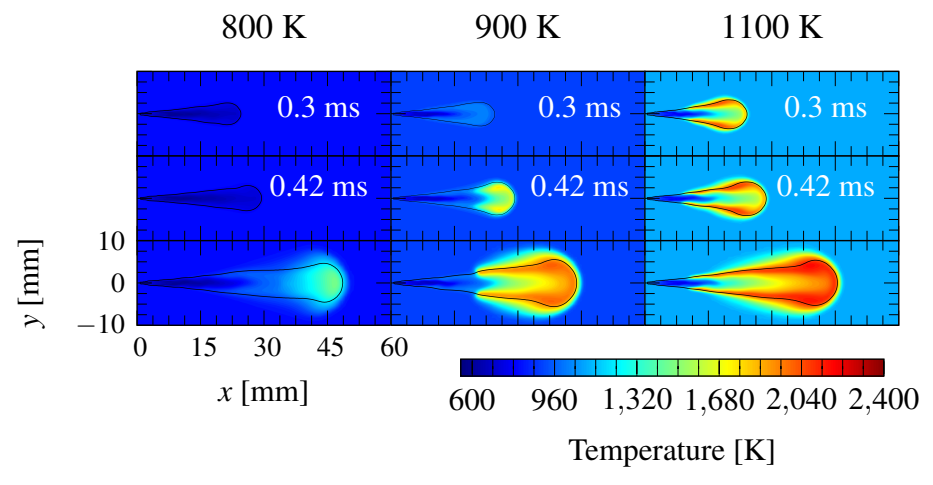

FIGURE 7: Instantaneous temperature fields of RANS simulations for various temperature "Spray A" conditions at $15 \%$ of fixed oxygen concentration. The time after start of injection is shown on the top right of each image. The times ASOI of the last line correspond respectively, from the left to the right, to 1.2 $\mathrm{ms}$ and $1.0 \mathrm{~ms}$ for the last two. The black line is the stoichiometric mixture fraction contour

Figures 7, 8 and 9, respectively, depict the variation of flame temperature, $\mathrm{CH}_{2} \mathrm{O}$ and $\mathrm{OH}$ mass fraction contours with changing ambient temperature between $800 \mathrm{~K}$ and $1100 \mathrm{~K}$. The black line in the temperature contour represents the isoline of the stoichiometric mixture fraction. Note that due to a comparatively extended ignition delay, the quasi-steady state time selected for the $800 \mathrm{~K}$ case in Figure 7 is $1.2 \mathrm{~ms}$ - as opposed to $1.0 \mathrm{~ms}$ for the $900 \mathrm{~K}$ and $1100 \mathrm{~K}$ cases. Note also that this extended ignition delay is consistent with the earlier discussion of the characteristics of the chemistry manifold, Figure 4. The temperature contours, Figure 7, show that the shorter ignition delay time at a higher ambient temperature leads to longer jet penetration and a smaller high temperature combustion spreading angle. The two stage ignition process is first detailed from the intermediate species, $\mathrm{CH}_{2} \mathrm{O}$, mass fraction contours, Figure 8, representing the first stage ignition, also known as the cool flame. Prior to the first stage ignition, the initial $\mathrm{CH}_{2} \mathrm{O}$ formation is seen at the periphery of the penetrating jet followed by convection to the jet centre. This is consistent with experimental observations of the characteristics of first stage ignition where heat release is observed at the jet periphery [?]. At the quasi-steady state condition, the highest concentrations of $\mathrm{CH}_{2} \mathrm{O}$ are found at the core of the jet (the outer regions having been consumed by high temperature second stage ignition processes). As the ambient tempera- 


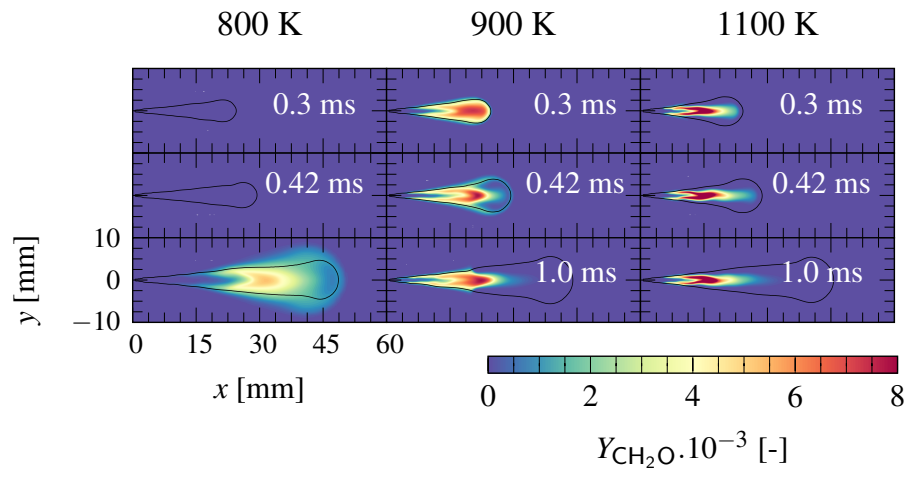

FIGURE 8: Instantaneous $\mathrm{CH}_{2} \mathrm{O}$ mass fraction fields of RANS simulations for various temperature "Spray A" conditions at $15 \%$ of fixed oxygen concentration. The time after start of injection is shown on the top right of each image with $1.2 \mathrm{~ms}$ corresponding to the last case of the $800 \mathrm{~K}$ simulation. The black line is the stoichiometric mixture fraction contour

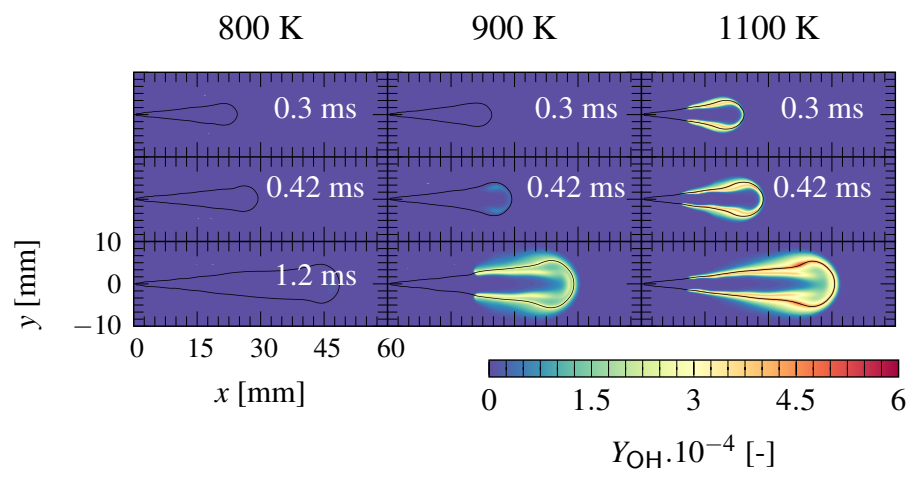

FIGURE 9: Instantaneous OH mass fraction fields of RANS simulations for various temperature "Spray A" conditions at $15 \%$ of fixed oxygen concentration. The time after start of injection is shown on the top right of each image with $1.0 \mathrm{~ms}$ corresponding to the last cases of the $900 \mathrm{~K}$ and the $1100 \mathrm{~K}$ simulations. The black line is the stoichiometric mixture fraction contour

ture is increased, the $\mathrm{CH}_{2} \mathrm{O}$ distribution becomes more compact, and the region of maximum $\mathrm{CH}_{2} \mathrm{O}$ concentration moves further upstream.

Hydroxyl $(\mathrm{OH})$ is chosen as the indicator of high temperature second stage ignition. The predicted $\mathrm{OH}$ mass fraction distributions, Figure 9 correspond well with both the temperature and the $\mathrm{CH}_{2} \mathrm{O}$ mass fraction contours. A significantly higher $\mathrm{OH}$ mass concentration is observed in higher temperature conditions. This is likely linked to the fact that, at lower temperatures conditions, the ignition delay time is prolonged, providing substantially higher mixing in the mixture. With a leaner mixture prior to second stage combustion, both flame temperature and the $\mathrm{OH}$ mass fraction are reduced. Overall, the effect of varying ambient temperature on the prediction of diesel spray auto-ignition and thereafter, flame propagation is well captured by the CSE-FGM model.

\section{Oxygen concentration sweep}

Following the temperature sweep described previously, the performance of the CSE-FGM combustion model was examined with respect to variations in oxygen concentration, keeping all other parameters fixed. The boundary conditions for these tests are given in Table 1, Tests II, V and VI refer. Figure 10 shows
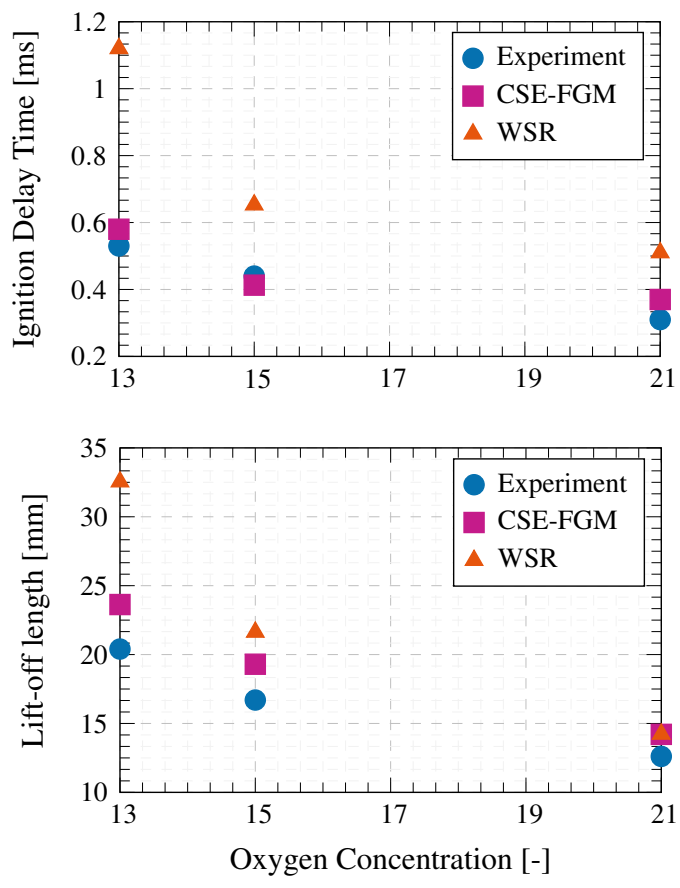

FIGURE 10: RANS CSE-FGM model results at $900 \mathrm{~K}$ with various ambient oxygen concentration conditions compared against the mean experimental results and the WSR model results for ignition delay time (top) and flame lift-off length (bottom)

the IDT and LOL simulations results compared to the mean experimental and the WSR model. An increase in oxygen concentration is seen to slightly accelerate the autoignition process, whereas the LOL is greatly shortened when oxygen concentration is high. 


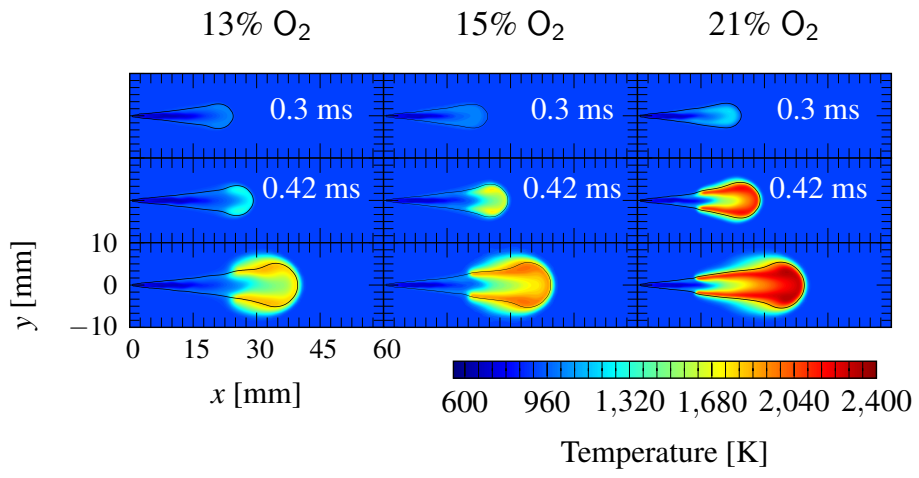

FIGURE 11: Instantaneous temperature fields of RANS simulations at $900 \mathrm{~K}$ "Spray A" conditions for various oxygen concentrations. The ASOI time is shown on the top right of each image with the last line corresponding to $0.8 \mathrm{~ms}$. The black line is the stoichiometric mixture fraction contour

Figures 11, 12 and 13, respectively, show the temperature, $\mathrm{CH}_{2} \mathrm{O}$ and $\mathrm{OH}$ mass fraction distributions at $900 \mathrm{~K}$ ambient temperature with various oxygen concentrations. The quasi-steady state time for all oxygen concentrations is chosen to be $0.8 \mathrm{~ms}$ after the start of the injection. From the $\mathrm{CH}_{2} \mathrm{O}$ plots, it is clear that a higher oxygen concentration leads to a faster first stage ignition where intermediate species are consumed earlier for second stage ignition. The position of the peak $\mathrm{CH}_{2} \mathrm{O}$ concentration is approximately constant at the downstream location of $15 \mathrm{~mm}$ at the highest oxygen concentration $(21 \%)$. Similar behaviour was observed in previous diesel spray simulations performed using other chemical mechanisms [?]. As shown in Figure 11, increasing the ambient oxygen concentration causes the onset of the high temperature reaction zone to be pushed upstream towards the injection nozzle - which is likely caused by the higher reactivity of the mixture when higher oxygen concentration boundary conditions are given. An increase of temperature at the tip of the spray is present for all flames in Figure 11; however, a clear increase in temperature is observed when oxygen concentration is increased. The variation of reactiveness of the flame can also be seen from the $\mathrm{OH}$ mass fraction distribution, Figure 13. A significant increase in absolute $\mathrm{OH}$ mass fraction is seen at $21 \%$ oxygen conditions where a high concentration of $\mathrm{OH}$ shifts to the injector tip, indicating a shorter LOL. It can be seen from these results that the CSE-FGM model is able to capture the subtle impact of changing species concentration on the ignition and flame characteristics.

\section{DISCUSSION - CSE-FGM RANS IN CONTEXT}

Previous sections of this work have presented results of an ambient temperature sweep and an oxygen concentration sweep

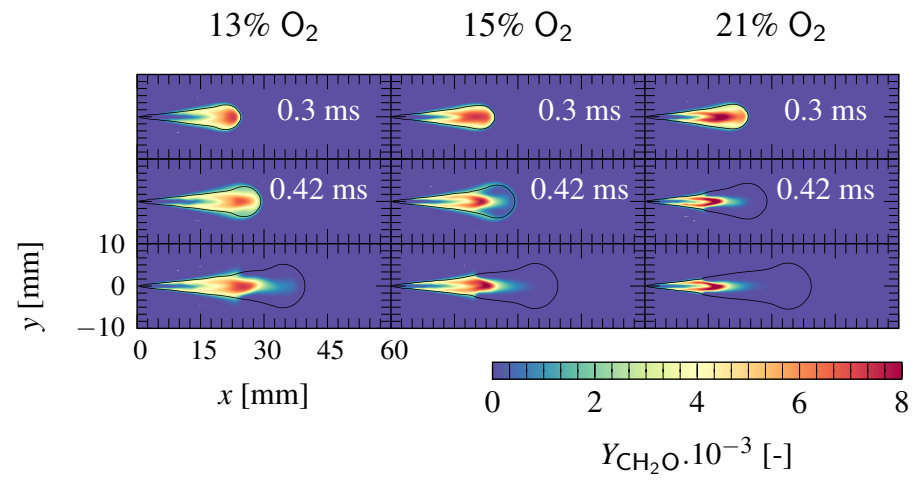

FIGURE 12: Instantaneous $\mathrm{CH}_{2} \mathrm{O}$ mass fraction fields of RANS simulations at $900 \mathrm{~K}$ "Spray A" conditions for various oxygen concentrations. The time after start of injection is shown on the top right of each image with the last line corresponding to 0.8 $\mathrm{ms}$. The black line is the stoichiometric mixture fraction contour

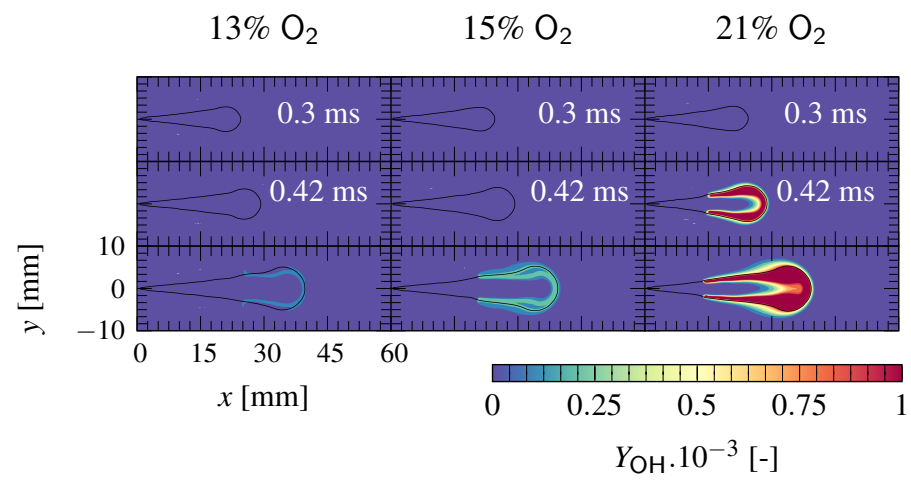

FIGURE 13: Instantaneous $\mathrm{OH}$ mass fraction fields of RANS simulations at $900 \mathrm{~K}$ "Spray A" conditions for various oxygen concentrations. The time after start of injection is shown on the top right of each image with the last line corresponding to 0.8 ms. The black line is the stoichiometric mixture fraction contour

\begin{tabular}{rcccc}
\hline Baseline & Experiment & LES Yao & LES Luo & RANS \\
\hline IDT $[\mathrm{ms}]$ & $0.40-0.44$ & 0.408 & 0.423 & 0.413 \\
LOL $[\mathrm{mm}]$ & $15.80-17.90$ & 18.08 & 22.16 & 19.31 \\
\hline
\end{tabular}

TABLE 3: Ignition delay time and flame lift-off length for ECN "Spray A" baseline condition. Experimental data repeated from [?]

of "Spray A" simulations using CSE-FGM within a RANS framework. In this section, the performance of the CSE-FGM RANS model under baseline conditions (Table 1, Test II) is compared with experimental data from the literature [?] and the results of a single CSE-FGM LES realisation [?] at $900 \mathrm{~K}$ from 


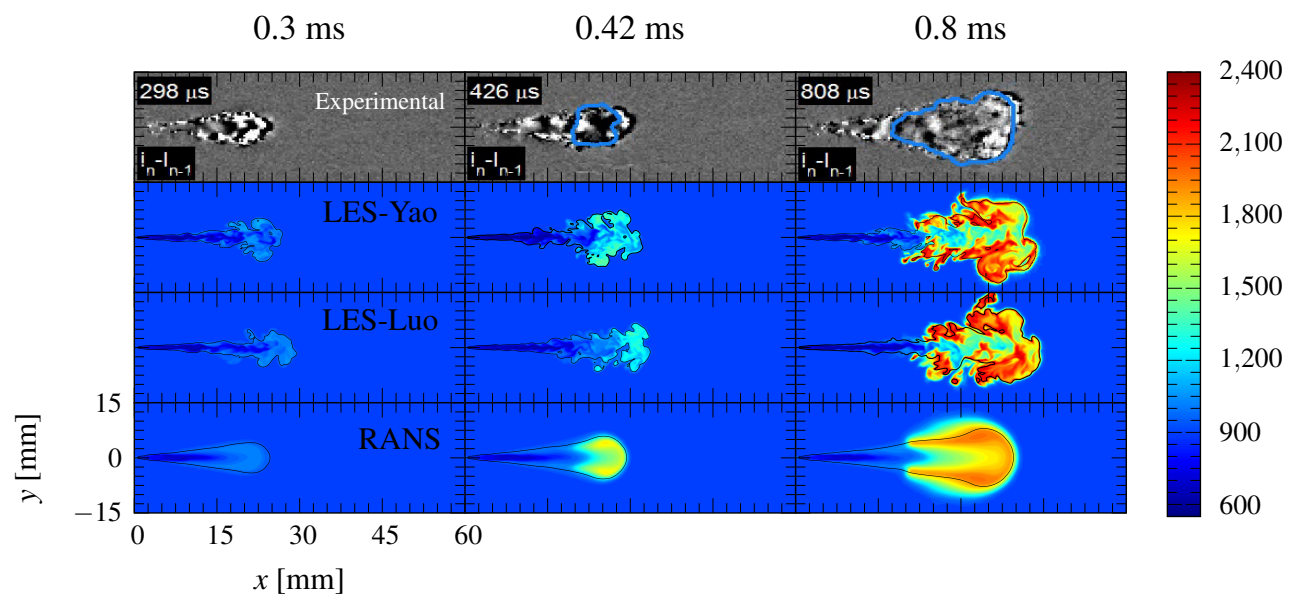

FIGURE 14: Schlieren image (top) and instantaneous temperature fields of LES and RANS simulations at $900 \mathrm{~K}$ "Spray A" baseline conditions. The time after start of injection is shown on the top left of each experiment images. The blue line in experimental study shows luminosity border. The black line is the stoichiometric mixture fraction contour. The experimental images are corrected by its background intensity $\left(I_{n}-I_{n-1}\right)$. The colourbar on the right corresponds to the temperature colourmap in Kelvin

two different chemical mechanisms - the Yao mechanism used in the RANS study, and the Luo mechanism (characterised by 106 species and 420 reactions [?]). Table 3 details the IDT and LOL results for all simulations. It can be seen that both the RANS and LES simulations are able to capture the combustion metrics to a high degree of accuracy. The IDT predicted by the RANS simulation falls between the LES simulations and is well within the range of the experimental data. Similarly, the RANS prediction of LOL falls between the two LES simulations results. In this case, all models slightly overpredict. Previous study has traced the overprediction for the LES simulation with the Luo mechanism to the manifold itself, which gives relatively lower source term values in regions associated with ignition and the intermediate stages of combustion stages [?].

The temperature contours of the RANS simulation are plotted against the LES Yao and LES Luo mechanisms and the schlieren experimental images in Figure 14. The border of the high-temperature chemiluminescence (blue line) is overlaid on the simultaneously-acquired schlieren images to help the visualization of the high temperature reaction. The details of image processing and the experimental set up can be found in [?]. The computed distributions from the RANS and both LES studies show good qualitative agreement with the experiments throughout the evolution of the flame - from ignition to quasi-steady state. As expected, there are significant temperature fluctuations present in the LES results compared to those of RANS simulations at the same timestep. Note also that the RANS flame structure is closer to the individual realisation of the flame captured in the experimental data than is the single LES realisation. As discussed in [?], this is also to be expected. For the RANS simulations the $\mathrm{Z}_{s t}$ line crosses the middle of the high temperature field, which is closer to the jet centerline, overlapping more of the high temperature region compared to LES simulation. In the LES, the $Z_{s t}$ line is located at the edge of the flame where high temperature reactions exist. The reason for this is that the LES simulation is a single realisation with spatial averaging, which results in a thin flame due to a very fine mesh. In contrast, RANS is an ensemble averaged simulation which thickens the flame and moves the $\mathrm{Z}_{s t}$ line to the centre of the flame.

\section{CONCLUSIONS}

Three-dimensional diesel "Spray A" simulations have been performed using a newly developed combustion model, CSEFGM, within a RANS turbulence framework. The model takes the first moment closure hypothesis from conditional moment closure methods; however, detailed chemistry is incorporated in a tabulated form giving greater flexibility. The simulations were carried out in the commercial code CONVERGE with unsteady flamelet solutions generated from CHEM1D using the a 54 species and 268 reactions mechanism of Yao.

A parametric study, considering a wide range of ambient temperatures and oxygen concentrations, was performed and the flame autoignition and propagation characteristics detailed. The CSE-FGM RANS model model successfully demonstrated the capability of realistically predicting the essential structure of the diesel-fueled transient spray combustion processes. The ignition delay time and flame lift-off length were predicted with good accuracy over the full range of ambient conditions examined. Analysis of the predicted temperature fields and intermediate species distributions also demonstrated the ability of the CSEFGM model to capture the two-stage ignition process present in 
diesel combustion.

The CSE-FGM RANS model results were also compared with the predictions of a WSR model. It is shown that the CSEFGM model leads to more accurate results, especially in low temperature conditions compared to the WSR model. This was attributed to the CSE-FGM model's inclusion of turbulence chemistry interactions.

Finally, a comparison between the results of the current CSE-FGM RANS study with a previous CSE-FGM LES study under baseline ECN "Spray A" conditions has demonstrated the consistency of the combustion model using different turbulence models. From a combustion modelling viewpoint, this study demonstrates that the CSE-FGM approach offers a feasible tool for detailed combustion analysis of diesel spray flames over a wide range of engine relevant conditions.

\section{ACKNOWLEDGEMENT}

The authors would like to thank EPSRC and China Oxford Scholarship Fund for the financial support. We would also like to thank Convergent Science Inc for CFD code access. Additionally, the authors would like to acknowledge the use of the University of Oxford Advanced Research Computing (ARC) facility in carrying out this work. 